EPJ Web of Conferences 47, 01006 (2013)

DOI: $10.1051 /$ epjconf/20134701006

(C) Owned by the authors, published by EDP Sciences, 2013

\title{
Periodic variability of spotted M dwarfs in WTS
}

\author{
N.T. Goulding ${ }^{1, a}$, J.R. Barnes ${ }^{1}$, D.J. Pinfield ${ }^{1}$, C. del Burgo 2,3, G. Kovács ${ }^{4}$, \\ J. Birkby ${ }^{5}$, S. Hodgkin ${ }^{4}$, S. Catalán ${ }^{1}$, B. Sipőcz ${ }^{1}$, H.R.A. Jones ${ }^{1}$, S.V. Jeffers ${ }^{6}$ \\ and S. Nefs ${ }^{5}$ \\ ${ }^{1}$ Centre for Astrophysics Research, Science \& Technology Research Institute, \\ University of Hertfordshire, Hatfield, Herts AL10 9AB, UK \\ ${ }^{2}$ Instituto Nacional de Astrofísica, Óptica y Electrónica (INAOE), Aptdo. Postal 51 y 216 , \\ 72000 Puebla, Pue., Mexico \\ ${ }^{3}$ UNINOVA-CA3, Campus da Caparica, Quinta da Torre, Monte de Caparica 2825-149, \\ Caparica, Portugal \\ ${ }^{4}$ Institute of Astronomy, University of Cambridge, Madingley Road, Cambridge CB3 OHA, UK \\ ${ }^{5}$ Leiden Observatory, Universiteit Leiden, PO Box 9513, 2300 RA Leiden, The Netherlands \\ ${ }^{6}$ Institut für Astrophysik, Friedrich-Hund-Platz 1, 37077 Göttingen, Germany
}

\begin{abstract}
We present an analysis of the photometric variability of M dwarfs in the WFCAM Transit Survey, selected from spectral types inferred by their WTS and SDSS colours, with periods detected using a LombScargle Periodogram Analisys. We estimate population membership of these objects from their tangential velocities and photometric parralaxes. Examples of $M$ dwarfs with variable light curve morphologuies are found. We discuss possible causes for this and make use of models of spotted stars in our interpretation of the results.
\end{abstract}

\section{INTRODUCTION}

The Wide Field Camera (WFCAM) Transit Survey (WTS) is a UK Infra-red Telescope (UKIRT) Campaign Survey comprising more than 100 nights worth of observations, compiled into light curves in the $J$ band supplemented with single, deep ZYJHK band exposures. The observation technique is described fully by Cappetta et al. [6], and the data reduction and light curve production was carried out by the Cambridge Astronomical Survey Unit (CASU) using a customised pipeline [6, 10]. These light curves permitting sensitivity to variability periods of up to tens of days.

The periodic variability in $\mathrm{M}$ dwarfs can result from the modulation of brightness by cool star spots. For solar-type stars the $\alpha-\Omega$ dynamo generated at the tachocline, the boundary between the convection and radiative zones [5]. However, stars later than M3.5 are fully convective, so no tachocline exists at which such a dynamo can be generated, yet stars of a later spectral type are still active and this activity may be dependent on an alternative form of dynamo $[4,15]$.

\section{SELECTION}

We made spectral classifications using colour-spectral type relations to identify the WTS M dwarfs. Convey et al. [8] find the location of main sequence stars across the Morgan-Keenan spectral types from

\footnotetext{
ae-mail: n.t.goulding@herts.ac.uk
}

This is an Open Access article distributed under the terms of the Creative Commons Attribution License 2.0, which permits unrestricted use, distribution, and reproduction in any medium, provided the original work is properly cited. 


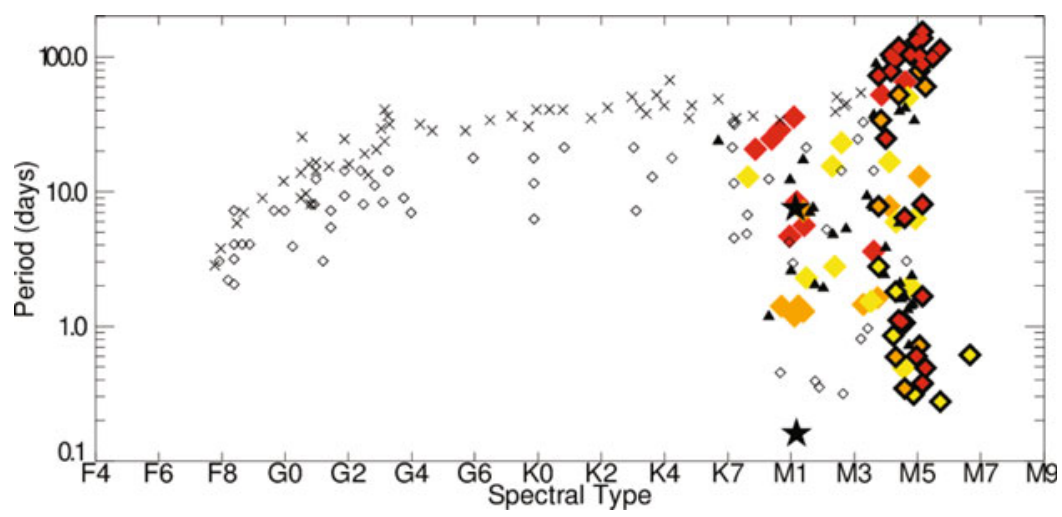

Figure 1. Periods of stars in our sample comared to literature sources, see text mfor colour definitions, stars with black borders from [11], crosses are young field stars, unfilled black diamonds are old field stars.

near infrared Two Micro All Sky Survey (2MASS) $J H K_{S}$ and visual Sloan Digital Sky Survey (SDSS) ugriz photometry. On SDSS/2MASS ugrizJHK $K_{S}$ colour-colour diagrams the loci of main sequence and giant stars are discernible enough to fit discrete tracks. We use the relative positions of each WTS star to these tracks to estimate whether a star is on the the main sequence or not. The spectral type can be estimated from the colour indices using the colour-spectral type relation presented by Convey et al. [8], and West et al. [19] who provide the relation extended to later spectral types. To assess the reliability of our classification, we applied the method to $M$ dwarfs of known spectral types from catalogues published by West et al. [19] and the match between our estimated spectral type and the spectroscopically determined spectral type follows a $1: 1$ relation with an error of approximately \pm 1 sub-type.

The Lomb-Scargle periodogram, which is optimised for finding sinusoidal shaped periodic signals $[13,16]$, is used to detect periodic signals in all the lightcuves of stars identified as M dwarfs. Sources of periodic variability other than that caused by spot modulation, such as binarity, are identified and eliminated from the sample. We discount all other potential identities for the variables as their colours are inconsistant with being identified as late-type, main sequence stars.

\section{RESULTS}

The variability search yields 71 detections of variable stars We make comparisons with rotationevolution studies, using tangential velocities $\left(v_{t}\right)$ obtained from SDSS proper motions and distance estimates from photometric parallaxes [3] as approximate tracers of population [e.g. 14]. We label stars with a $v_{t} \gtrsim 30 \mathrm{kms}^{-1}$ as likely old disk stars (red diamonds in Fig. 1), and those with a $v_{t}<15$ as young disk stars (yellow diamonds). Other stars are marked with orange diamonds. Two of the faintest stars in our sample are found to have $v_{t}=309 \pm 35 \mathrm{kms}^{-1}$ and $v_{t}=814 \pm 79 \mathrm{kms}^{-1}$ (marked with black stars), consistent with being members of the halo. We find our old disk stars are primarily slower rotators, corroborating the results and collated data of Kiraga \& Stępień [12], and the later MV stars in Irwin et al. [11], who find a greater number of slow rotators amongst the old disk stars, and also find young disk stars occupying the full range of detected periods. We find the majority our 'old' stars at periods $>20$ days, in agreement with their conclusion of a more rapid spin down time for more massive, partially radiative stars. Although we do find 5 early MV stars with periods $<10$ days, this may be as a result of the crude method of assuming an age from $v_{\text {tan }}$ that does not take into account the full space velocity of the star, and these stars may in fact be members of the younger population, as early $\mathrm{M}$ types only remain active for $<2$ Gyr [18]. 


\section{Hot Planets and Cool Stars}
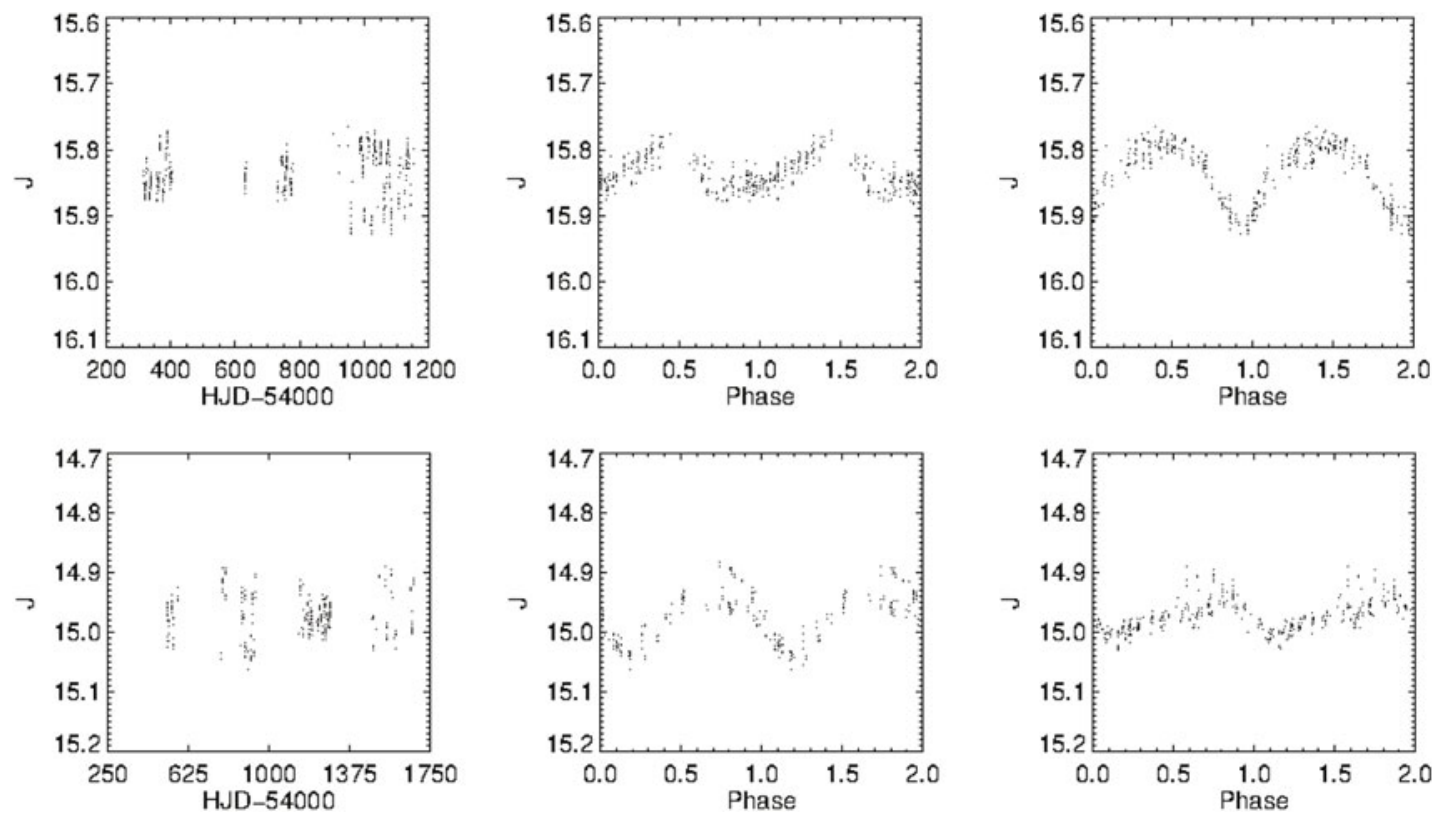

Figure 2. The upper plots show light curves of the stars 19c_2_05428 and folded about the same period of 20.68 days; the centre panel showing observations at HJD $<54900$ and the panel on the left with observations at HJD > 54900. The lower plots show and 07e_2_02466, folded about the same period of 12.86 days; the centre panel showing observations at HJD $<55000$ and the panel on the left with observations at HJD $>55000$.

We find some evidence for a change in spot coverage was found in one star with an indeterminate spectral type in the 17hr field, 17d_1_06032, and in the stars 07e_2_02466 and 19c_2_05428 (Fig. 2). In the former, no significant peak in the periodogram, performed over the entire series of observations, corresponded to an obvious periodicity in folded light curves, though the most significant peak in the periodogram for only the later $50 \%$ of the observations did correspond to a period for which a folded light curve displayed obvious periodic behavior. In the latter two a strong peak corresponding to the period is found in their periodograms although the amplitude of the variation changes over timescale of months, perhaps similarly indicative in a change in the spottedness of the stars. The changes in both instances happened over a time scale of $<100$ days. In both 17d_1_06032 and 19c_2_05428 however, the change in amplitude was dichotomous and does not appear to vary much once the new amplitude is established, whereas in 07e_2_02466 the amplitude varies over hundreds of days.

One instance of a $\Delta J>0.2$ flaring event is serendipitously discovered from the M4V( \pm 1$)$ star 19d_1_12692. This magnitude of flare is in contrast to the results of Tofflemire et al. [17] who expect to find flaring events in the $J$ band occurring on mmag scales, and would transform to a $u$-band response of $>\Delta 6$, which by extrapolation of the frequencies of flaring events per magnitude found by Davenport et al. [9] would be expected to be observed less than once a year.

\section{DISCUSSION}

We have detected $\mathrm{M}$ dwarfs with periods between hours and several weeks, with the largest period found at 90.3 days. For comparison, periods up to 154 days have been found for other $\mathrm{M}$ dwarfs Benedict et al. [2], Irwin et al. [11], Kiraga \& Stępień [12]. Whilst the WTS periodic variable M dwarf sample produced no convincing evidence of periodicity on these time scales, a large number of periods $>80$ days were found in the periodicity search and subsequently rejected when no sinusoidal variability 


\section{EPJ Web of Conferences}

was seen. Indeed it is not expected that all spot coverage on a star will induce a periodic variability in brightness if the spot coverage is not sufficiently inhomogeneous to invoke a detectable difference in brightness as the star rotates, or if the spot coverage changes in morphology at shorter time scales than the rotation period, although even in such a case a rotation period should be found by a Lomb-Scargle periodogram.

A spot model is used to generate a range of scenarios against which the results presented here can be compared. The simulations were generated by the Doppler Tomography of Stars code (DoTS) [7] that can produce synthetic light curves at a chosen wavelength (13000 , corresponding approximately to the $J$ band midpoint) for a range of photospheric and spot temperatures ( $T_{p}, T_{s}$ respectively) and spot coverage models. The spot coverage models are identical to those used by Barnes et al. [1], including seven models in total covering solar minimal and maximum coverage to more extreme cases for active stars [see Table 1, 1].

From the simulations, synthetic light curves are obtained giving a range of peak to peak amplitudes for each scenario of spot coverage, against which the variable stars in the sample can be compared in order to place limits on the spot coverage of each star. These simulations place some limits on the spot coverage over the variable $\mathrm{M}$ dwarfs found here that suggest a star spot coverage modeled at $18 \%$ and $48 \%$ for lower $T_{s} / T_{p}$ ratios and a lower coverage model at $6.1 \%$ for the larger $T_{S} / T_{p}=0.6$ model.

Further simulations making use of evolving spot coverages, including the growth and decay of star spots and differential rotation will allow for the study of the long term conherence of light curve modulation by spots in variable stars and will further inform the prospects of transit detection for planets orbiting highly active spotted stars.

\section{CONCLUSION}

From a sub-sample of variable objects are selected using a Lomb-Scargle periodogram, 71 variable rotating MV stars are found with a range of periods from 0.16 to 90.3 days and amplitudes from 0.009 to 0.115 in the $J$ band. We infer population membership from tangential velocities and find our results to be in agreement with previous such studies. Using a lightcurve synthesis code we find that these stars may have a high degree of spottedness, of the order of 10 per cent surface coverage or more, in some cases $\gtrsim 50$ per cent, at least at time scales of $<20$ days, and we estimated a fraction for these variable $\mathrm{M}$ dwarfs of at least of 1 per cent from the most complete subsamples. These results indicate that transit surveys carried out in the $J$ band may be less susceptible to the effect of spot induced variability in photometric observations, as our simulations suggest they are predisposed to only detecting the variability of the most active of M dwarfs. One example of a particular large flare in the $J$ band is serendipitously found. We also find evidence for evolving spot morphologies in the form of light curve amplitudes varying over periods of months in three of stars. In one late type dwarf of unknown spectral type periodic variability was found to switch on after months of inactivity.

\section{References}

[1] Barnes, J. R., Jeffers, S. V., \& Jones, H. R. A. 2011, MNRAS, 412, 1599

[2] Benedict, G. F., McArthur, B., Nelan, E., et al. 1998, AJ, 116, 429

[3] Bochanski, J. J., Hawley, S. L., \& West, A. A. 2011, AJ, 141, 98

[4] Brown, B. P., Browning, M. K., Brun, A. S., Miesch, M. S., \& Toomre, J. 2008, ApJ, 689, 1354

[5] Browning, M. K., Basri, G., Marcy, G. W., West, A. A., \& Zhang, J. 2010, AJ, 139, 504

[6] Cappetta, M., Saglia, R. P., Birkby, J. L., Koppenhoefer, J., Pinfield, D. J., Hodgkin, S., et al. 2012, MNRAS, 427, 1877-1890

[7] Collier Cameron, A. 1997, MNRAS, 287, 556

[8] Covey, K. R. et al. 2007, Astron. J., 134, 2398

[9] Davenport, J. R. A., Becker, A. C., Kowalski, A. F., et al. 2012, ApJ, 748, 58 


\section{Hot Planets and Cool Stars}

[10] Irwin, J., Irwin, M., Aigrain, S., et al. 2007, MNRAS, 375, 1449

[11] Irwin, J., Berta, Z. K., Burke, C. J., et al. 2011, ApJ, 727, 56

[12] Kiraga, M. \& Stępień, K. 2007, Acta Astron., 57, 149

[13] Lomb, N. R. 1976, APSS, 39, 447

[14] Reiners, A. \& Basri, G. 2008, ApJ, 684, 1390

[15] Rockenfeller, B., Bailer-Jones, C. A. L., \& Mundt, R. 2006, A\&A, 448, 111

[16] Scargle, J. D. 1982, ApJ, 263, 835

[17] Tofflemire, B. M., Wisniewski, J. P., Kowalski, A. F., et al. 2012, AJ, 143, 12

[18] West, A. A., Hawley, S. L., Bochanski, J. J., et al. 2008, AJ, 135, 785

[19] West, A. A., Morgan, D. P., Bochanski, J. J., et al. 2011, AJ, 141, 97 